\title{
Knowledge on occupational exposures and Post Exposure Prophylaxis (PEP) of blood-borne infections among dental surgeons in four hospitals in Colombo; a cross sectional study
}

\author{
Dr AAGH Anupama ${ }^{1}$, Dr RPNK Rajapaksha², Dr PAG Navaratne ${ }^{3}$, \\ Dr RMAS Rathnayaka ${ }^{4}$, Dr HMAH Karunaratne ${ }^{5}$
}

\begin{abstract}
Introduction: Dental surgeons are at higher risk of getting exposed to blood-borne pathogens like Human Immunodeficiency Virus (HIV), Hepatitis B Virus (HBV) and Hepatitis C Virus (HCV). Therefore, dental surgeons should have updated knowledge on Occupational Exposures (OE) and Post Exposure Prophylaxis (PEP) for blood-borne infections.

Objective: To assess the level of knowledge on Occupational Exposures (OE) and Post Exposure Prophylaxis (PEP) for blood-borne viral infections among government dental surgeons, in four hospital units in the Colombo district.

Method: Cross sectional descriptive study was carried out using a self-administered questionnaire among all dental surgeons (143) in dental units of four teaching hospitals in the Colombo district. namely; National Dental (Teaching) Hospital, Colombo, Lady Ridgeway Hospital, Colombo South Teaching Hospital, Kalubowila, and Institute of Oral Health, Maharagama, in 2019.

Results: Almost three fourth (74.3\%) of dental surgeons had experienced OEs. Needle pricks identified as the common occupational injury. About $55 \%$ dental surgeons knew about the immediate measures required to be taken after an OE but, only $7 \%$ had gone through the PEP counseling process. Majority of dental surgeons $87.1 \%$ had been vaccinated for HBV. However, only $66 \%$ knew about their antibody titres. The majority of dental surgeons had better knowledge on PEP for HIV than PEP for HBV and HCV.
\end{abstract}

Conclusions: Occupational exposures commonly occur among dental surgeons. More PEP training \& awareness programs are required for dental surgeons to improve their safety.

Keywords: Dental Surgeons, Occupational Exposures, Post Exposure Prophylaxis.

Authors: ${ }^{1}$ Dr AAGH Anupama (BDS, postgraduate trainee, Oral and Maxillofacial Surgery), National Dental (Teaching) Hospital, Colombo. ${ }^{2}$ Dr RPN. Rajapaksha (BDS, postgraduate trainee, Oral and Maxillofacial Surgery), National Dental (Teaching) Hospital, Colombo, ${ }^{3}$ Dr PAG Navaratna( BDS,MS. Colombo, FDSRCS.Ed, FDSRCS.Lon); Consultant in Oral and Maxillofacial surgery, National Dental (Teaching) Hospital, Colombo. ${ }^{4}$ Dr RMAS Rathnayaka (BDS,MS.Colombo,FFDRCS.I), Consultant in Oral and Maxillofacial surgery, National Dental (Teaching) Hospital, Colombo, ${ }^{5}$ Dr H.M.A.H. Karunaratne (MBBS,Pg.DipVen,MD); National STD/AIDS Control Programme, Colombo.

Corresponding author: Dr AAGH Anupama, Email: harindione@gmail.com, (Dhttps://orcid.org/00000003-0821-1286

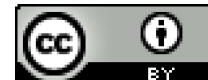

Acknowledgment: All the participants of the study, Conflict of interest: no conflict t of interest, Funding: No funding support for this study

Originality, previous publications, or presentations: This is an original work not published anywhere, presented at $24^{\text {th }}$ Annual Scientific Sessions of Sri Lanka College of Sexual Health and HIV Medicine 2019 and $16^{\text {th }}$ Annual Scientific Sessions of Sri Lanka Association of Oral and Maxillofacial Surgeons 2019. 


\section{Full article}

\section{Introduction}

Health care workers (HCWs) have potentially high exposure to blood, body fluid and other potentially infectious materials (OPIM) and the incidence is rising worldwide.(3) Occupational exposures to infectious material can result from percutaneous or mucocutaneous injuries, exposure to mucous membranes and nonintact skin. (1)

Annually 66,000 Hepatitis B, 16,000 Hepatitis $C$, and 200-500 HIV infections are developed among HCWs, due to exposure to infectious material.(4) The most common form of Occupational Exposure to blood is needle prick injury. (2)

Among the HCWs, dental practitioners have high exposure risk to infectious materials. They are exposed to blood and body fluid (saliva contaminated with blood). Further, dental surgeons are prone to exposures due to frequent use of sharp instruments and highspeed rotary instruments that produce contaminated aerosols. (2) Hence, measures should be taken to prevent or minimize OEs among dental surgeons, but the knowledge on PEP is equally important in a situation when there is an unexpected OE.

There is limited information on the incidence and risk of exposure to Blood-Borne Infections (BBI) among dental surgeons in Sri Lanka basically due to a lack of researches conducted in this regard. $(5,6)$ This information is important in designing effective PEP related intervention programs.

This study was planned to assess the level of knowledge on OE and PEP for blood-borne viral infections among government dental surgeons, in the Colombo district.

\section{Method}

A descriptive cross-sectional study was carried out in dental units of four teaching hospitals in Colombo district, namely; National Dental (Teaching) Hospital, Colombo; Lady Ridgeway Hospital for Children, Colombo 10; Colombo
South Teaching Hospital, Kalubowila, and Institute of Oral Health, Maharagama, in 2019.

The study involved consultants, postgraduate trainees, and grade dental surgeons working at specialized dental units and Out-Patient Departments (OPD) in dental units of the above hospitals. All the dental surgeons in respective units were invited to the study and 143 participated.

A self-administered questionnaire was used for the data collection. The questionnaire assessed their level of knowledge on blood-borne viral infections, the incidence of OEs, and their PEP awareness. The principal investigator and coinvestigators were available for clarifications during the data collection process. Data were analyzed using SPSS 21 . Quantitative data were analyzed with regards to measures of central tendency and dispersion and qualitative data were analyzed with regards to proportions.

\section{Results}

\section{Socio-demographic characteristics.}

The majority $(69.9 \%)$ of the dental surgeons were females and most $(58.7 \%)$ were grade dental surgeons with a basic degree. Only $31.5 \%$ conducted private clinics. The sociodemographic profile is shown in table 1.

Table 1: Socio-demographic profile

\begin{tabular}{|l|l|r|r|}
\hline \multicolumn{2}{|l|}{$\begin{array}{l}\text { Socio-demographic } \\
\text { characteristic }\end{array}$} & No. & $\%$ \\
\hline \multirow{4}{*}{ Gender } & Male & 42 & 29.4 \\
\cline { 2 - 4 } & Female & 100 & 69.9 \\
\cline { 2 - 4 } & Total & $142^{\mathrm{a}}$ & 100 \\
\hline \multirow{4}{*}{$\begin{array}{l}\text { Education } \\
\text { level }\end{array}$} & BDS & 84 & 58.7 \\
\cline { 2 - 4 } & BDS+Diploma & 32 & 22.4 \\
\cline { 2 - 4 } & PG trainee & 21 & 14.7 \\
\cline { 2 - 4 } & MD & 6 & 4.2 \\
\cline { 2 - 4 } Institution & Total & 143 & 100 \\
\cline { 2 - 4 } & Dental OPD & 17 & 11.9 \\
\cline { 2 - 4 } & OMF unit & 52 & 36.4 \\
\cline { 2 - 4 } & Restorative unit & 33 & 23.1 \\
\cline { 2 - 4 } & Orthodontic unit & 27 & 18.9 \\
\cline { 2 - 4 } & $\begin{array}{l}\text { Community } \\
\text { dental unit }\end{array}$ & 11 & 7.7 \\
\cline { 2 - 4 } & $\begin{array}{l}\text { Oral pathology } \\
\text { unit }\end{array}$ & 3 & 2.1 \\
\cline { 2 - 4 } & Total & 143 & 100 \\
\hline \multirow{5}{*}{$\begin{array}{l}\text { Place of } \\
\text { practice }\end{array}$} & $\begin{array}{l}\text { Only } \\
\text { hospital }\end{array}$ & 97 & 67.8 \\
\hline \multirow{4}{*}{ Govt } & & \\
\hline
\end{tabular}




\begin{tabular}{|c|c|c|c|}
\hline & $\begin{array}{l}\text { Govt. hospital + } \\
\text { Private clinic }\end{array}$ & 45 & 31.5 \\
\hline & Total & $142^{\mathrm{a}}$ & 100 \\
\hline
\end{tabular}

\section{Occupational exposure}

The majority $(74.3 \%)$ of dental surgeons have had OE to blood or body fluids. Commonly occurring exposures were needle pricks (28\%), injuries from sharp instruments (24\%), and splash to eyes (28\%). Those three types account for $80 \%$ of OE s.

Graph 1:Type of Occupational Exposure (OE)

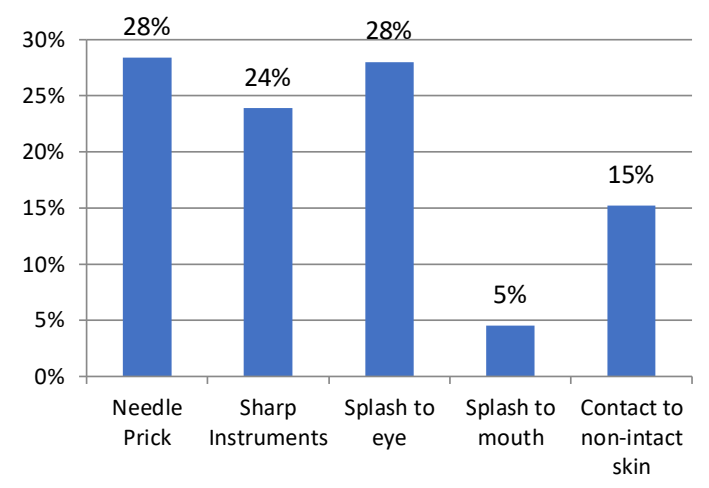

\section{Awareness of HIV PEP}

Among the 143 dental surgeons, $72 \%(\mathrm{~N}=104)$ had heard about PEP and only $7 \%$ participated in at least one PEP awareness program. Almost one-fifth of the sample (21\%) neither heard about nor participated in PEP awareness programs.

Just over half (55\%) of the sample knew about the immediate measures that should be taken after an OE. The majority (64.3\%) believed that PEP reduces the likelihood of getting HIV/AIDS but, only $35.7 \%$ knew about points where they can access PEP against HIV. Only $25 \%$ knew where to access HBV PEP services.

Only $38.6 \%$ knew that for the best outcome, critical timing to start PEP for HIV is within 2 hours of the exposure. Only $17.3 \%$ knew about the duration of PEP for HIV.

Only $7 \%$ of the sample had gone to formal PEP counselling.
Graph 2: PEP awareness among dental surgeons

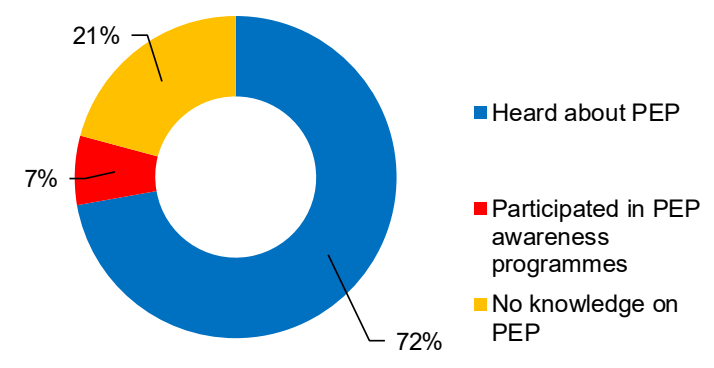

Only 30\% knew about the government PEP circular and $50 \%$ of dental surgeons do not know functioning protocols upon PEP.

\section{Knowledge of hepatitis B and Hepatitis C}

All the study participants knew about the availability of the Hepatitis B vaccine. Even though the majority ( $87.1 \%$ ) of dental surgeons had been vaccinated for HBV, only $59.1 \%$ of them knew about their antibody titre. Only $25.9 \%$ knew the rate of transmission of HBV when compared to HCV and HIV.

One third $(35.3 \%)$ of dental surgeons have treated for Hepatitis $C$ patients but they had very limited knowledge of HCV transmission and preventive measures. Nearly three-fourth of dental surgeons didn't know about the risk of getting Hepatitis $\mathrm{C}$ after contamination with infected blood.

only $28.7 \%$ of the participants knew that there is no vaccine for Hepatitis $C$.

\section{Discussion}

According to this study, $74.3 \%$ of dental surgeons ever had exposure to infectious materials when providing oral care and about $8.6 \%$ of them could not recall an event. This emphasizes the underreporting of OEs among the group.

Needle pricks are the commonest type of exposures among dental surgeons which is a serious risk among dental professionals. This happens mostly when using a syringe and recapping the needle. (7) According to the current study, one fourth of dental surgeons have sustained needle pricks / sharp injuries and mucous splash. 
Transmission of Hepatitis B depends on the type of injury (Percutaneous or mucosal) and the concentration of virions. Hepatitis B antigen shows high titre in blood and serous fluids other than saliva, (1) Hence, there is a high risk of transmission of Hepatitis $B$ even among the dental surgeons because of high rate of needle handling.

There are guidelines and instructions to prevent Hepatitis B transmission in dental practice. $(8,9)$ All the HCWs who are at risk of exposure to blood-born infections are given Hepatitis $B$ vaccination and the response is checked 1-2 months after the final dose. If the anti-Hbs titre is $>10 \mathrm{mlU} / \mathrm{ml}$, it shows the immunity. Hepatitis $B$ vaccination is repeated If the antibody titre is lower. (10)

The HCW who have received Hepatitis B vaccination and have developed immunity shows virtually no risk of infection. But for a susceptible person, the risk ranges 6\%-30\% from a susceptible individual. (11) Therefore measures should be taken to minimize the risk of getting Hepatitis B.

The current study shows the majority of dental surgeons (87.1\%) have been vaccinated for Hepatitis B and $66 \%$ of them know about their antibody titre. Further, $75 \%$ of them did not know where to access HBV PEP. This emphasizes that majority of dental surgeons did not know the measures to be taken following exposure.

Hepatitis $\mathrm{C}$ is a blood-borne pathogen which is having an average risk of infection after exposure to infected blood. There is no vaccine for Hepatitis $C$ and there is no post-exposure prophylaxis treatment to prevent getting disease after exposure. (12) This study shows only about $26 \%$ of dental surgeons have fundamental knowledge on Hepatitis $C$, hence dental surgeons need an extensive awareness on Hepatitis C.

The average risk of HIV, following percutaneous exposure to infected blood, is low $(0.3 \%)$. There are many interventions targeted towards occupational exposures and PEP including PEP management guidelines for HIV. $(11,13,14,15)$ In Sri Lanka, a circular has been released by the ministry of health, regarding PEP following OE. (15) In this study, only $30 \%$ of dental surgeons knew about the above circular. Further, $72 \%$ of dental surgeons have heard about PEP and 55\% of dental surgeons knew about the immediate measures, to be taken following OE. Only half $(50 \%)$ of the dental surgeons didn't show any knowledge of PEP protocols in their institutions.

Even though the majority has sustained $\mathrm{OE}$ in their working place, staggeringly, Only $7 \%$ of them had gone for PEP counseling as well as only $7 \%$ have participated in at least one PEP awareness program. Moreover, $64.3 \%$ of dental surgeons believe PEP reduces the likelihood of getting HIV/AIDS. This highlights the necessity of more and more PEP awareness and related programs targeting dental surgeons in Sri Lanka.

According to literature, the possibility of getting seroconversion following proper PEP is nearly zero(16). So it is very beneficial to have a piece of sound knowledge on PEP, as well as immediate commencement of PEP following risky exposure and proper follow up to minimize the risk of getting infected with Hepatitis B, C, or HIV. Hence, it is of utmost importance to increase dental surgeons' awareness of OEs and PEP while improving PEP service uptake by developing effective PEP programs.

\section{Conclusions}

Occupational exposures to infectious materials are common among the dental surgeons and needle pricks, injuries by sharp instruments and eye splashes are responsible for $80 \%$ of OE events. Under such circumstances, the existing level of knowledge and awareness of OE and PEP is inadequate among Dental Surgeons to minimize the risk of getting infected with Hepatitis B, C, or HIV.

\section{References}

1. Beltrami EM, Williams IANT, Shapiro CN. Risk and Management of Blood-Borne Infections in Health Care Workers. 2000;13(3):385-407.

2. Wicker S, Rabenau HF. Occupational exposures to bloodborne viruses among German dental professionals and students in a clinical setting. 2010;77-83. 
3. Ward P, Hartle A. UK healthcare workers infected with blood-borne viruses: Guidance on risk, transmission, surveillance, and management. Contin Educ Anaesthesia, Crit Care Pain. 2015;15(2):103-8.

4. Denić LM, Ostrić I, Pavlović A, Dimitra KO. Knowledge and occupational exposure to blood and body fluids among health care workers and medical students. Acta Chir lugosl. 2012;59(1):71-5.

5. Rajapakshe RWKMD, Dayananda LP, Dharmawardhana NMNJK, Perera PJPA. Knowledge of occupational exposures and Post Exposure Prophylaxis (PEP) for HIV among Health Care Workers (HCW) in Kegalle District, Sri Lanka Full article. 2017;31-7.

6. Karunaratne HMAH, Dissanayake $\mathrm{Y}$, Karawita DA. Knowledge and practices on HIV post-exposure prophylaxis (HIV PEP) among medical and dental doctors in the Teaching Hospital, Anuradhapura.

7. Shah SM, Merchant AT, Dosman JA. Percutaneous injuries among dental professionals in Washington State. 2006;5:2-6.

8. Academy TA. The prevention of hepatitis Larry I. Lutwick, MD Abstract B transmission in dental practice Dental Care Transmission of HBV. 1982;4(4):296-9.

9. Cleveland JL, Gray SK, Harte JA, Robison VA, Moorman AC, Gooch BF. Transmission of bloodborne pathogens in US dental health care settings: 2016 update. J Am Dent Assoc [Internet]. 2016;147(9):729-38. Available from: http://dx.doi.org/10.1016/j.adaj.2016.03.020

10. CDC. Which workers in the health care setting need the hepatitis B vaccine? Occup Health (Auckl) [Internet]. 1991;43(10):1-2. Available from: http://www.who.int/occupational_health/activities /3hepatiti.pdf

11. Centers for Disease Control and Prevention. What Healthcare Personnel Need to Know. Centers Dis Control Prev [Internet]. 2003;1200:10. Available from: http://bookstore.phf.org

12. Hcv T, Hcv T, Rna HCV, Ag HC V, Hcv C, Rna HC V. Hepatitis $C$ virus: epidemiology and transmission risks. 2016;(May):77-82.

13. Kuhar DT, Henderson DK, Struble KA, Heneine W, Thomas V, Cheever LW, et al. US PHS Guidelines for the Management of Occupational Exposures to HIV Updated U.S. Public Health Service Guidelines for the Management of Occupational Exposures to HIV and Recommendations for Postexposure Prophylaxis Update: Interim Statement Regarding Po. Natl Cent Emerg Zoonotic Infect Dis. 2015;1-48.

14. Shugars DC, Ph.D., Strauss RP, Ph D. Counseling, Testing, and Referral : Survey of U. S. Dental Schools. 2002; (October):1169-77.

15. Circular PEP HIV.pdf.

16. RRS. The efficacy of post-exposure prophylaxis (PEP) for HIV Key Take-Home Messages The Issue and Why It 's Important. Ontario HIV Treat Netw. 2019;337(March):2. 